\title{
Cinco métodos de manejo de plántulas para la inducción de brotación de basales en la variedad de rosa Blush de los Andes (Rosa spp)
}

\author{
Five methods for the management of plantules in order to induce \\ the growing of basals of a variety of Andean Blush rose (Rose spp.)
}

\author{
Rosita Espinoza Gavilánez* y Hugo Vinueza Mejía
}

Universidad Politécnica Salesiana, Carrera de Ingeniería Agropecuaria, Cayambe, Ecuador.

* Autor para correspondencia: gespinozag@ups.edu.ec, rosita5_espinoza@yahoo.es

Manuscrito recibido el II de septiembre de 2009. Aceptado, tras revisión, el 19 de noviembre de 2009.

\begin{abstract}
Resumen
La presente investigación muestra cinco métodos para la inducción de brotación en las plántulas de la variedad de rosa Blush de los Andes (Rosa spp). Los tratamientos empleados fueron: primero, agobio de la plántula con semiquiebre, previa formación de escobilla; segundo, agobio de la plántula por arqueo, previa formación de escobilla; tercero, cosecha de la primera flor en punto de corte ruso; cuarto, agobio de la plántula por arqueo, previo descabece del primer botón floral en punto garbanzo; $y$ por último, descabece del primer botón floral en punto de corte ruso más desyeme de brotes axilares. Se evaluó semana a semana el número de basales brotados por planta de cada tratamiento, la longitud en centímetros alcanzada por cada basal, el calibre en milímetros alcanzado por cada uno de los tallos, y se determinó el número de días a corte (ciclo del cultivo), desde la siembra de la plántula hasta la cosecha en punto ruso; y desde la implementación del tratamiento hasta la cosecha en el mismo punto de cada tallo basal evaluado. En cuanto al número de basales por planta, no se encontró diferencia significativa; en cuanto la longitud de basales en centímetros, el mejor tratamiento fue el segundo con una longitud promedio de $97,21 \mathrm{~cm}$ por tallo, al igual que en el calibre (diámetro) con un promedio de 6,39 mm por tallo, en la variable ciclo de cultivo desde la 'siembra', los mejores resultados se encontraron en los dos primeros tratamientos, en la misma variable pero desde la ejecución de los tratamientos, los mejores resultados se presentaron en el quinto caso.
\end{abstract}

Palabras clave: Agobio, punto de corte, basal, ciclo de cultivo, plántula, escobilla.

\begin{abstract}
The present study shows five techniques to induce the growing of plantules of a variety of Andean Blush rose (Rosa spp.). The techniques employed were as follows: in the first place, suffocation of plantules by semi-snapping prior to the growing of escobilla; second, suffocation of plantules by bowing, prior to the growing of escobilla; third, harvest of the first flower by the Russian cutting technique; fourth, suffocation of plantules by bowing prior to removing the first bud off in the manner of a chickpea; and fifth, removing the first flower bud by the Russian cutting technique plus removing the leaf buds from armpit shoots. The number of basals grown per treated plant was evaluated on a weekly basis, including the length in centimeters of each basal, the width in centimeters of each stalk, as well as the number of days (cultivation cycle) from planting of the plantules to the harvest at the same point of each basal stalk evaluated. As far as the number of basals per plant, no significant difference was found; as for the length of basals in centimeters, the best technique was the second, giving an average length of $97.21 \mathrm{~cm}$ per stalk, also for the width (diameter) with an average of $6.39 \mathrm{~mm}$ per stalk; as for the variable "cultivation cycle from planting", the best results were obtained from the two first treatments; however, for the same variable but since the application of the treatments, the best results were obtained from the fifth technique.
\end{abstract}

Keywords: suffocation, cutting point, basal, cultivation cycle, plantules, escobilla.

Forma sugerida de citar:

Espinoza Gavilanez, R. y Vinueza Mejía, H. 2009. Cinco métodos de manejo de plántulas para la inducción de brotación de basales en la variedad de rosa Blush de los Andes (Rosa spp). La Granja. 10 (2): Pp 47-55. ISSN: I390-3799

La Granja 10(2): 47-55. 2009.

(C) 2009, Universidad Politécnica Salesiana, Ecuador. 


\section{Introducción}

La explotación del cultivo de rosas como tal, empezó en el Ecuador en 1983 con una superficie de 2 ha, la misma que ha ido incrementándose tanto, que en la actualidad se tiene un aproximado de 2.749 ha de flores cultivadas, de las cuales el $63,50 \%$ corresponde a las rosas para corte (Rizzo Pastor, P., 200I).

Como cultivo intensivo las rosas para corte abarcan grandes extensiones de tierra, las mismas que cada vez son más escasas, razón que hace que los productores se vean en la necesidad de optimizar las mismas, implementando técnicas de cultivo que mejoren la producción. En los momentos actuales debido al incremento de precios de los insumos básicos utilizados en el cultivo de flores como los fertilizantes y pesticidas, a lo que se suma la elevación de salarios destinados a la mano de obra, se hace necesario adoptar medidas que equilibren los costos de producción con los beneficios obtenidos del cultivo. Entre las medidas que se toman se puede mencionar: el uso de calefacción nocturna en los invernaderos; aumento de la eficacia y eficiencia en el cultivo, para reducir el porcentaje de flor local nacional dada de baja por problemas fitosanitarios o por no cumplir con los estándares de calidad; la utilización de hormonas (auxinas, citoquininas, giberelinas) para acelerar la cosecha; lograr que plántulas recién plantadas se produzcan en el menor tiempo posible (formación de plantas) e inducir una mayor brotación de basales en el menor tiempo posible, entre los principales.

Considerando que el uso de calefacción en los invernaderos puede resultar muy costoso y el empleo de productos químicos para acelerar la apertura de botones puede ser contraproducente, se hace necesario realizar una investigación que permita determinar qué método(s) de manejo de plántulas de rosa, específicamente de la variedad Blush de los Andes (Rosa spp) (Ver Figura I), es el más adecuado para inducir una mayor brotación de basales en el menor tiempo posible, para formar una planta que asegure el mejoramiento de la productividad en cultivo.

Es sumamente importante asegurar el número de yemas basales, su número depende de la edad y la posición de la yema y, generalmente, sólo las dos yemas inferiores entre las yemas potenciales producen brotes basales. Si más tarde se desarrolla un tercer brote o más, provendrá de la yema axilar de uno de los dos brotes ya crecidos, diferentes factores afectan la formación y calidad de los brotes basales (López et al., 2007).

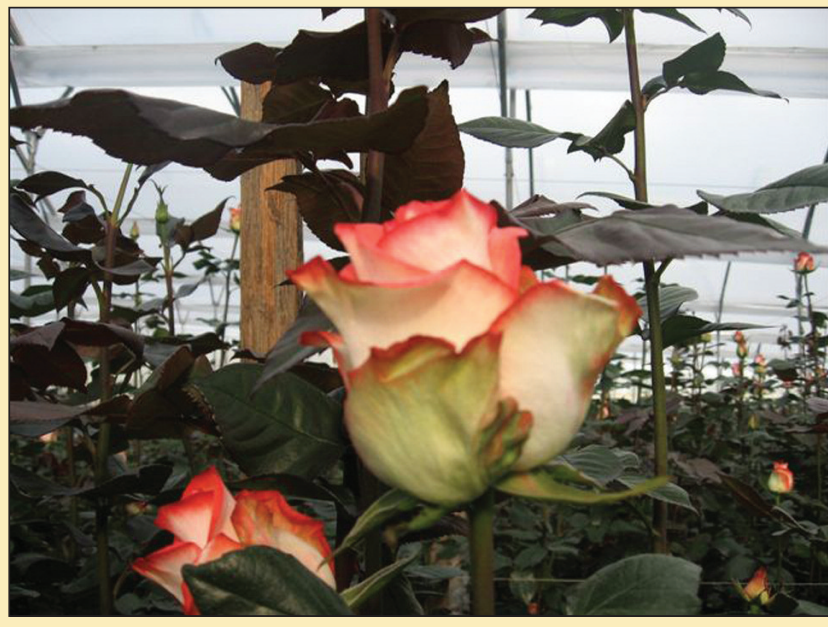

Figura I.Variedad 'Blush de los Andes'.

Fuente: Hugo Vinueza Mejía

\section{Materiales y métodos}

Se utilizaron plántulas de la variedad de rosa 'Blush de los Andes' (Rosa spp), injertadas bajo el método de 'stenting', elegido por su rapidez de propagación en el que una rosa para corte se injerta sobre un patrón sin enraizar (Duys y Schouten, 200 I), las mismas que fueron plantadas en la empresa florícola Rose Connection, en el cantón Cayambe provincia de Pichincha, Ecuador.

El diseño utilizado en esta investigación fue un Diseño Cuadrado Latino (DCL) $5 \times 5$, ya que se estudió cinco manejos distintos de plántulas para inducir la brotación de los basales.

El objeto de estudio para esta investigación fue el medir la eficiencia de cinco diferentes tipos de manejo de plántulas (propagadas con injerto de aproximación) para inducir la brotación de basales en la variedad mencionada.

Las variables en estudio de esta investigación incluyeron: número de basales, longitud de basales, calibre (diámetro) de basales y días a la cosecha (ciclo de cultivo), con el objetivo de mejorar los procesos.

\section{I Preparación y escogitamiento de la muestra}

En primer lugar, se procedió a la preparación del suelo que consiste en la arada, rastrada e incorporación de correctivos entre los cuales tenemos: cascarilla de arroz, cascarilla de café y cascajo. Hecho esto, se procedió a desinfectar el suelo con el ingrediente activo 
Dazomet a razón de 40 gramos por $\mathrm{m}^{2}$, el cual se lo dejó actuar por el lapso de 21 días incorporado al suelo y cubierto con plástico para lograr una mayor eficacia del producto.

Posterior a la desinfección, se realizó un riego profundo para garantizar que no exista fitotoxicidad en las plántulas a ser sembradas. Seguido a la labor anterior se efectuó nuevamente un volteo del suelo utilizando arado y rastra, para a continuación de estas labores proceder a nivelar, preparar camas y sembrar (Ver Figura 2).

Una vez listo el suelo, se construyeron camas de $35,5 \mathrm{~m}$ de largo por $0,80 \mathrm{~m}$ de ancho, entre las cuales existe un camino de $0,54 \mathrm{~m}$ de ancho. Las plántulas fueron sembradas en dos hileras con disposición de tres bolillo y una distancia entre plántula de 17,6 $\mathrm{cm}$. Cada cama tiene nueve cuadros, $y$ se sembraron un promedio de 403 plántulas por cama.

Para la investigación se seleccionaron cinco camas sembradas (2.015 plántulas aproximadamente, ver Figura 3), con un promedio de 45 plántulas por cuadro y un total de 45 cuadros.

Según normas del diseño utilizado se realizó la delimitación del efecto de borde, para lo cual se decidió no tomar en cuenta a los dos cuadros iniciales y dos cuadros finales de cada cama en estudio, quedando de esta manera un total de 25 cuadros y 1.125 plántulas para el estudio.

Se instaló el Diseño Cuadrado Latino DCL $(5 \times$ 5) para la investigación en el cultivo, para lo cual se procedió al sorteo de los tratamientos, así como el sorteo de las columnas e hileras respectivamente. Se etiquetaron las plántulas para ser evaluadas ( 10 por parcela neta), previo un sorteo, una vez que se delimitó el efecto de borde de cada tratamiento ( 10 plántulas al inicio y 10 plántulas al final de cada tratamiento).

Una vez escogida la muestra de estudio, la medición se llevó a cabo cada 7 días, a partir de la ejecución de los tratamientos; mientras que la evaluación de los tratamientos se realizó hasta que los basales brotados de cada planta tratada llegaron a punto ruso.

La fertilización y lámina de riego se realizó bajo condiciones de la finca y el control de plagas $y$ enfermedades fue el mismo que la finca utiliza para el resto de variedades.

Posteriormente, se realizó el respectivo análisis económico de los tratamientos.

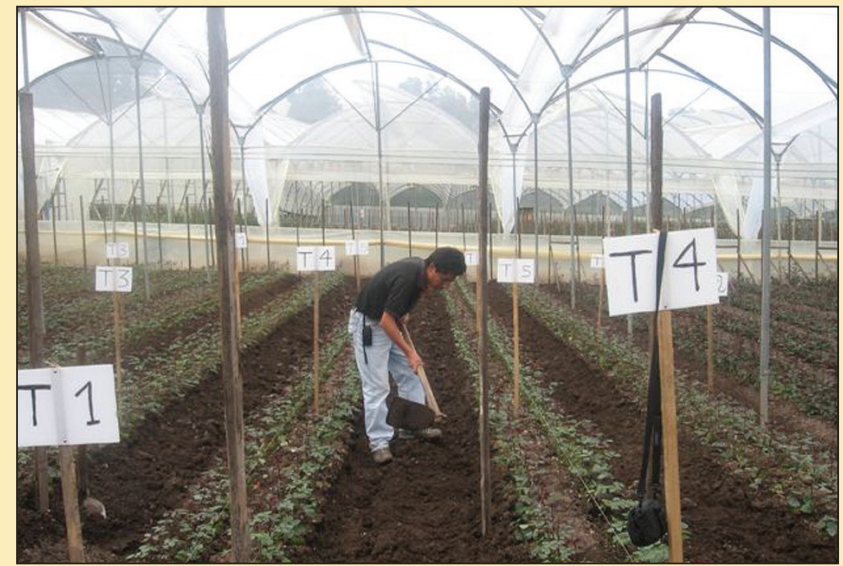

Figura 2. Manejo del experimento.

Fuente: Hugo Vinueza Mejía.

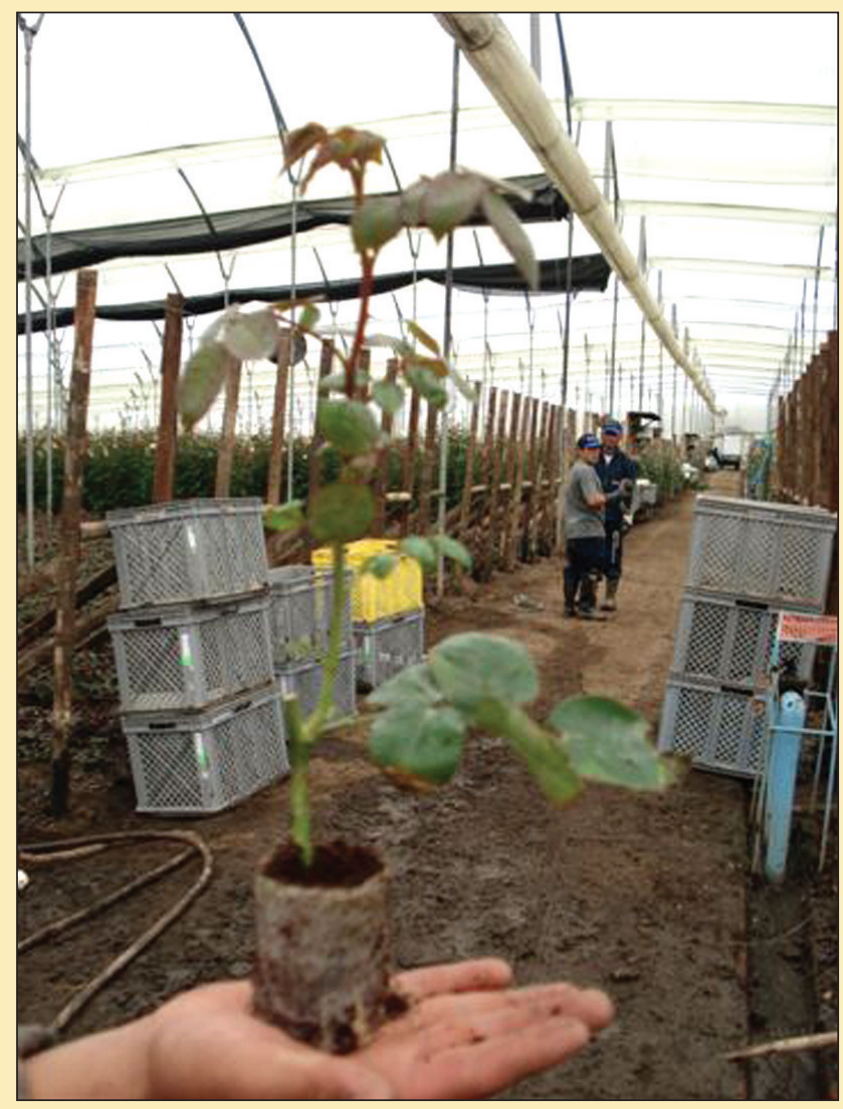

Figura 3. Plántulas de la variedad Blush de los Andes (Rosa $s p p)$.

Fuente: Hugo Vinueza Mejía. 


\subsection{Aplicación de los tratamientos}

\subsection{Tratamiento I.Agobio de la plántula con semiquiebre, previa formación de escobilla}

Una vez que las plántulas se encontraron estableci-

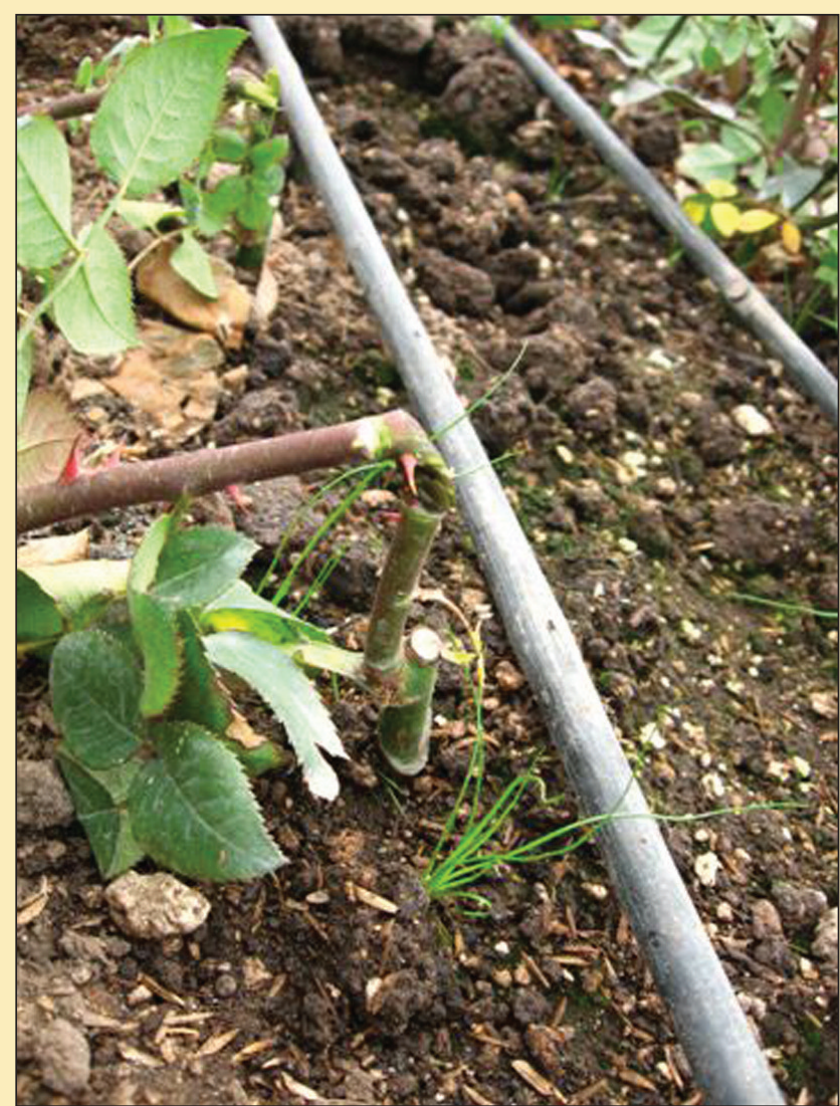

Figura 4. Agobio de plántulas con semiquiebre.

Fuente: Hugo Vinueza Mejía.

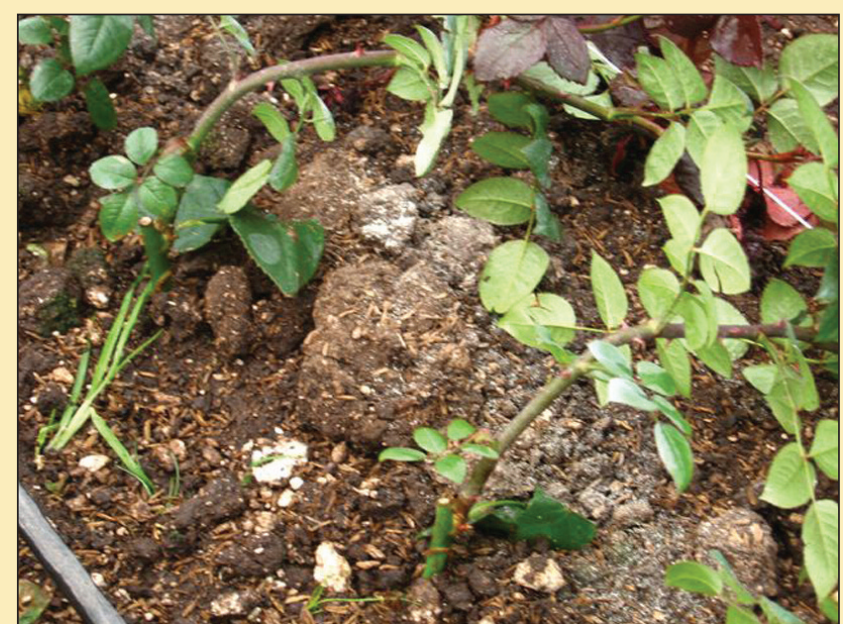

Figura 5. Agobio de plántulas por arqueo previa formación de la escobilla.

Fuente: Hugo Vinueza Mejía. das en el cultivo, se procedió a la formación de las 'escobillas', para lo cual se efectuó un descabece del primer botón floral en punto arroz de cada plántula evaluada, el mismo que se realizó tan pronto como la plántula mostró el punto establecido ( 8 días a partir de la siembra). Seguido a esto se esperó la emisión de yemas axilares, a las cuales se les realizó la misma labor del descabece en punto arroz tan pronto como apareció este estadio, lo cual favoreció la formación de las escobillas; una vez formadas las escobillas con alrededor de 4-5 brotes axilares descabezados (lo cual se obtuvo a los 35 días luego de realizado el descabece del primer tallo en punto arroz), se procedió al agobio utilizando la técnica conocida como 'semiquiebre' (Ver Figura 4), ya que se procede a romper parte del tejido del tallo con el empleo de un alicate para facilitar el agobio y evitar el uso de la piola para sostener a las plántulas, este semiquiebre se realizó a $5 \mathrm{~cm}$ de altura desde la base del brote principal.

\subsubsection{Tratamiento 2. Agobio de la plántula por arqueo, previa formación de escobilla}

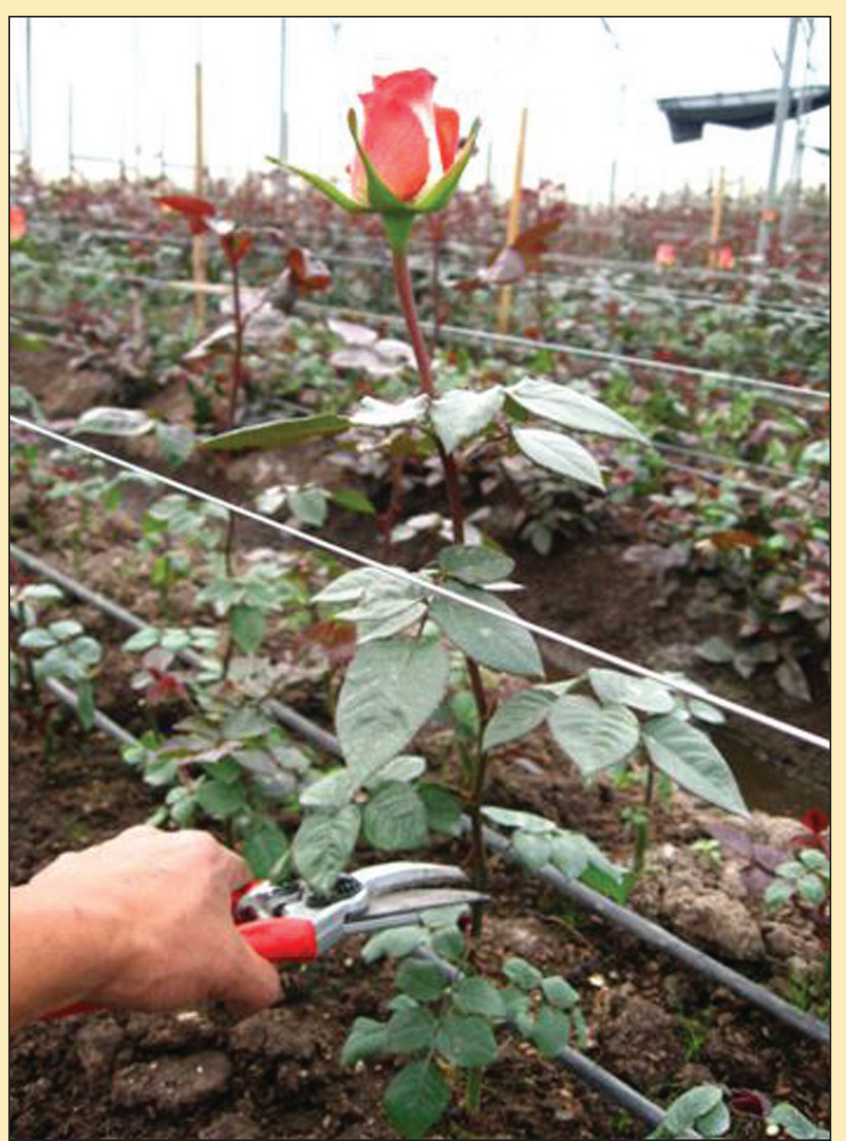

Figura 6. Cosecha de la primera flor en punto ruso. Fuente: Hugo Vinueza Mejía. 


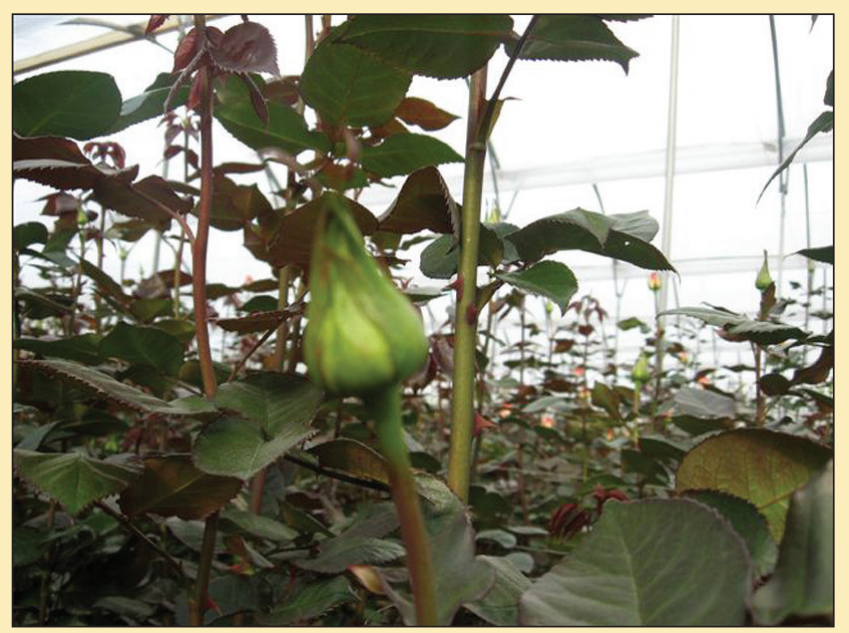

Figura 7. Punto garbanzo.

Fuente: Hugo Vinueza Mejía.

En la primera parte se procedió de la misma manera que en el tratamiento uno, es decir primero se formó las 'escobillas', luego de lo cual se realizó el agobio por arqueo (Ver Figura 5), realizado inclinando las plántulas hacia la parte de afuera de la camas (caminos) y utilizando líneas de piola para mantener esta inclinación.

\subsubsection{Tratamiento 3. Cosecha de la primera flor en punto de corte ruso}

Para este tratamiento, una vez establecidas la plántulas, se procedió al desyeme de brotes axilares cada 7 días (tiempo establecido en la finca para esta labor), hasta una altura de $12 \mathrm{~cm}$ desde la base del tallo (Ver Figura 6), donde una vez que los botones principales llegaron a su punto de corte (punto ruso), se procedió a cosecharlos (42 días contados a partir de la siembra).

\subsubsection{Tratamiento 4. Agobio de la plántula por arqueo, previo descabece del primer botón floral en punto garbanzo}

Una vez establecidas la plántulas en el sitio definitivo (unidad experimental), se procedió al desbrote de yemas axilares cada 7 días (tiempo establecido en la finca para esta labor), hasta que las plántulas

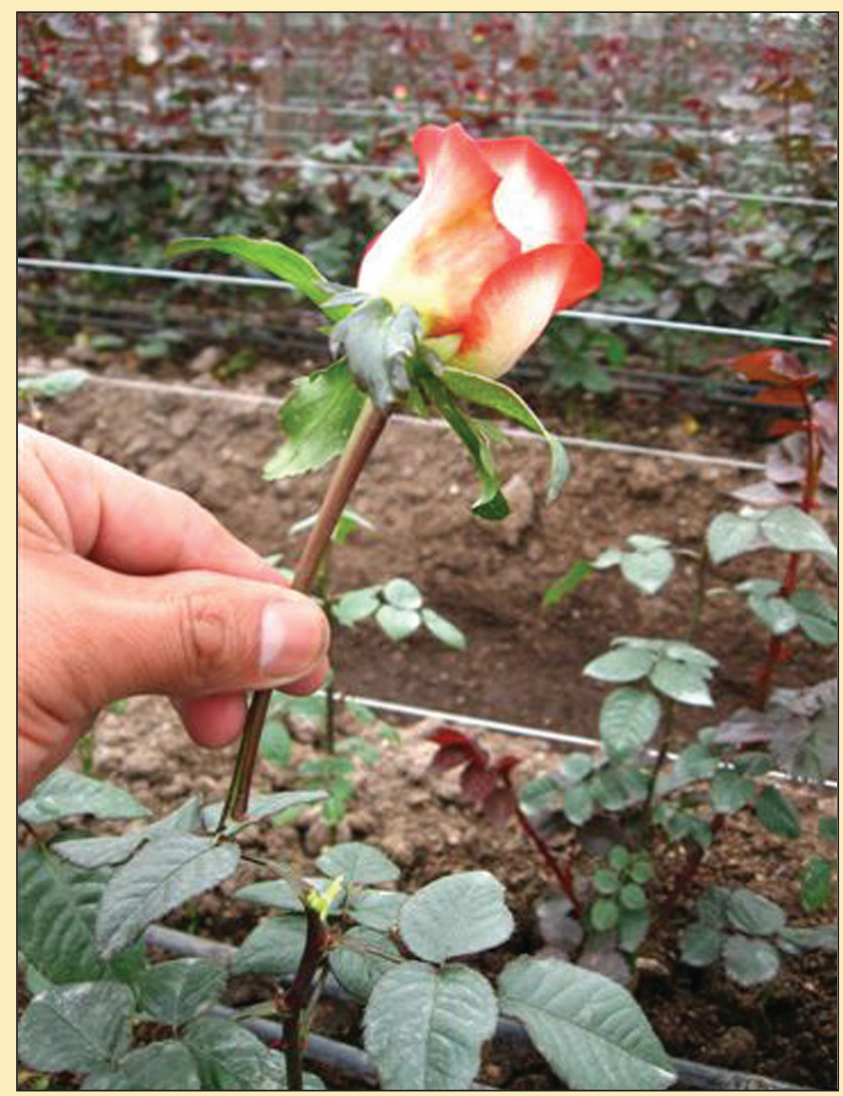

Figura 8. Descabece del botón en punto ruso. Fuente: Hugo Vinueza Mejía.

mostraron sus primeros botones florales, los mismos que cuando llegaron a punto garbanzo, presentado en la Figura 7 (25 días a partir de la siembra), fueron descabezados, seguido del agobio por arqueo, el cual se realizó a los 7 días después de realizado el descabece (32 días a partir de la siembra).

\subsubsection{Tratamiento 5. Descabece del primer bo- tón floral en punto de corte ruso más desye- me de brotes axilares.}

En este tratamiento, una vez establecida la plántula, se procedió al desyeme de brotes axilares cada 7 días (establecido por la finca). Una vez que el botón principal llegó a su punto de corte (punto ruso) se procedió a descabezarlo (42 días contados a partir de la siembra) y se continuó desyemando las yemas axilares del tallo hasta que la planta empezó a emitir sus basales, tal como se presenta en la Figura 8. 


\section{Resultados y discusión}

\section{I Número de basales}

Tabla I. Incremento en el número de basales en los diferentes tratamientos cada 7 días.

\begin{tabular}{|c|c|c|c|c|c|c|c|c|c|c|}
\hline \multirow{3}{*}{ 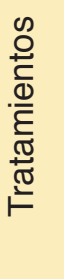 } & \multicolumn{6}{|c|}{ Número de basales por tratamiento } & \multirow{3}{*}{$\begin{array}{c}\text { Promedio de basales } \\
\text { por planta por } \\
\text { tratamiento }\end{array}$} & \multirow{3}{*}{ 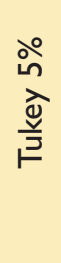 } & \multirow{3}{*}{ 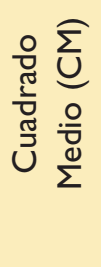 } & \multirow{3}{*}{$\stackrel{\dot{u}}{\text { ن }}$} \\
\hline & \multicolumn{6}{|c|}{ Días } & & & & \\
\hline & 7 & 14 & 21 & 28 & 35 & 42 & & & & \\
\hline TI & 23,00 & 37,00 & 55,00 & 83,00 & 85,00 & & $\mathrm{I}, 70$ & a & NS & $|0,6|$ \\
\hline $\mathrm{T} 2$ & 9,00 & 11,00 & 26,00 & 63,00 & 71,00 & 79,00 & 1,58 & a & & \\
\hline T3 & 17,00 & 23,00 & 36,00 & 66,00 & 71,00 & 76,00 & 1,52 & a & & \\
\hline T4 & 45,00 & 61,00 & 71,00 & & & & $\mathrm{I}, 42$ & a & & \\
\hline T5 & 27,00 & 48,00 & 69,00 & & & & $\mathrm{I}, 38$ & a & & \\
\hline
\end{tabular}

Con los resultados obtenidos en la Tabla I, se encuentra que entre el número de basales promedio por plántula y por tratamiento, no existe significancia, es decir que estadísticamente todos los tratamientos son iguales, ya que cada uno de ellos cum-

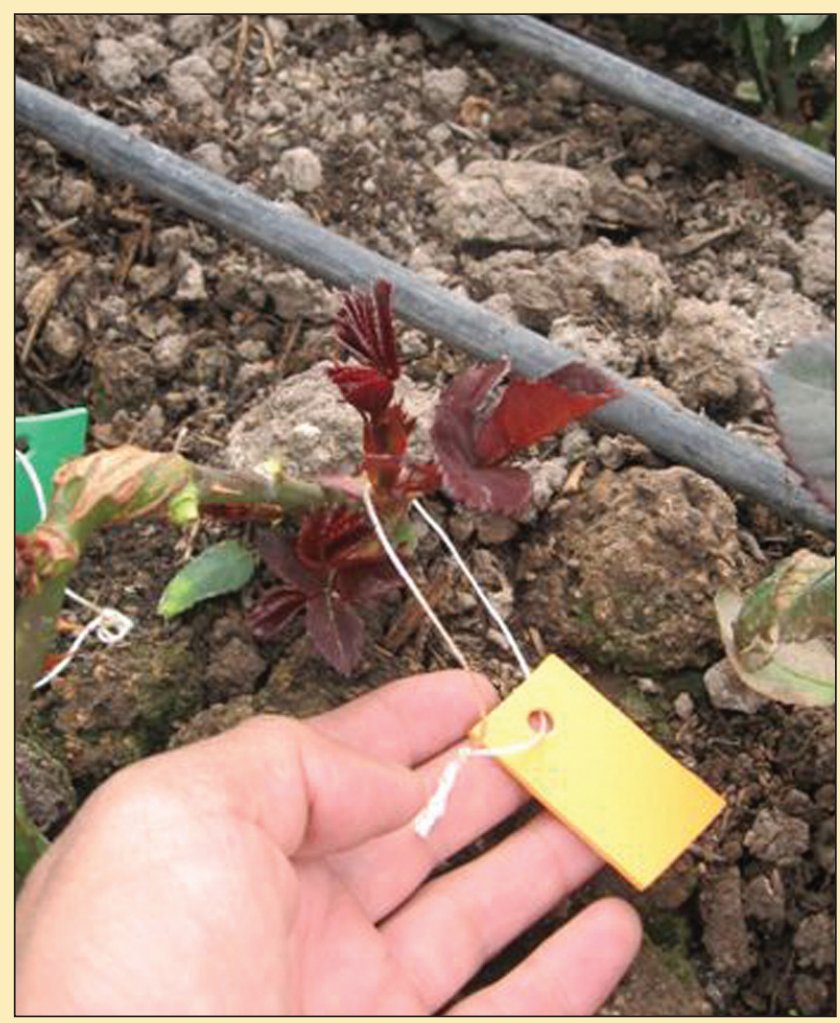

Figura 9. Basales brotados.

Fuente: Hugo Vinueza Mejía. ple con la labor de eliminar la dominancia apical en los tallos de la plántula de rosa, con lo cual se induce a la brotación de las yemas bajeras (Ver Figura 9), que en este caso son las que dan origen a los brotes basales.

\subsection{Longitud de basales}

Tabla 2.Comportamiento de los basales en la variable longitud, medidos cada 7 días.

\begin{tabular}{|c|c|c|c|c|}
\hline 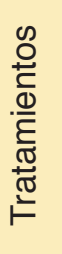 & $\begin{array}{l}\text { Longitud promedio } \\
\text { en centímetros por } \\
\text { tallo por tratamiento }\end{array}$ & $\begin{array}{l}\text { ㅇํ } \\
\text { बे } \\
\text { 를 }\end{array}$ & 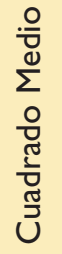 & ن \\
\hline T2 & 97,21 & $\mathrm{a}$ & & \\
\hline T4 & 94,37 & $a b$ & & \\
\hline T5 & 89,37 & b c & $* *$ & 2,81 \\
\hline TI & 85,47 & c d & & \\
\hline T3 & 83,45 & D & & \\
\hline
\end{tabular}

Se encontró en la Tabla 2 que existe alta significancia estadística para tratamientos, es decir que los tratamientos son diferentes unos de otros.

$\mathrm{Al}$ analizar el comportamiento de estos resultados, para la variable longitud de basales en centímetros, el mejor comportamiento lo presentan los 2 
tratamientos en los cuales se realizó el agobio por arqueo, es decir en el tratamiento 2 y 4 , ya que el tratamiento uno (agobio de la plántula con semiquiebre, previa formación de escobilla) se encuentra compartiendo el rango (cd) en cuarto lugar de importancia, descartándose que no es sólo el efecto de escobilla el que llevó al tratamiento 2 al primer lugar de importancia.

\subsection{Calibre (diámetro) de basales}

Tabla 3. Calibre de basales en milímetros, medidos cada 7 días.

\begin{tabular}{|c|c|c|c|c|}
\hline 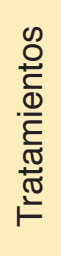 & $\begin{array}{c}\text { Calibre promedio } \\
\text { en milímetros por } \\
\text { tallo por } \\
\text { tratamiento }\end{array}$ & 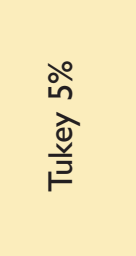 & 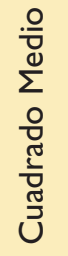 & $\overrightarrow{\mathrm{u}}$ \\
\hline T2 & 6,39 & A & $* *$ & 8,32 \\
\hline T4 & 5,59 & $a b$ & & \\
\hline TI & 5,27 & b c & & \\
\hline T5 & 4,62 & cd & & \\
\hline T3 & 4,22 & d & & \\
\hline
\end{tabular}

Del análisis obtenido de la Tabla 3, se observa que existe alta significancia estadística para tratamientos, es decir que todos los tratamientos son distintos unos de otros.

En el primer rango (a) se encuentra el tratamiento 2 (agobio de la plántula por arqueo, previa formación de escobilla) con un promedio de $6,39 \mathrm{~mm}$ de diámetro.

Compartiendo el rango a y $b$ se encuentra el tratamiento cuatro (agobio de la plántula por arqueo, previo descabece del primer botón floral en punto garbanzo) con un promedio de $5,59 \mathrm{~mm}$.

El tratamiento uno (agobio de la plántula con semiquiebre, previa formación de escobilla) con 5,27 $\mathrm{mm}$ de promedio y el tratamiento 5 (descabece del primer botón floral en punto de corte ruso más desyeme de brotes axilares) con un promedio de 4,62 $\mathrm{mm}$ se encuentran compartiendo los rangos (bc) y (cd), respectivamente.

En el último rango (d) se encuentra el tratamiento 3 (cosecha de la primera flor en punto de corte ruso) con 4,22 mm promedio de diámetro.

\subsection{Días a la cosecha (ciclo de cultivo)}

Tabla 4. Días a la cosecha (ciclo del cultivo) desde la 'siembra' de las plántulas de todos los tratamientos.

\begin{tabular}{|c|c|c|c|c|}
\hline 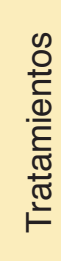 & $\begin{array}{c}\text { Promedio en días } \\
\text { a la cosecha } \\
\text { por tratamiento }\end{array}$ & 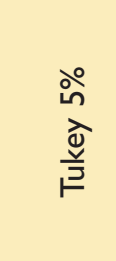 & 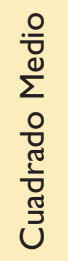 & $\overrightarrow{\mathrm{u}}$ \\
\hline T5 & 127,48 & A & $* *$ & 2,56 \\
\hline T4 & 129,88 & $a b$ & & \\
\hline T3 & 136,52 & B & & \\
\hline T2 & $|49,4|$ & C & & \\
\hline TI & 152,76 & C & & \\
\hline
\end{tabular}

Evaluados los tratamientos podemos observar que existe una alta significancia estadística entre los mismos con un coeficiente de variación de 2,56 que da confiabilidad a los resultados.

Realizada la prueba de Tukey al $5 \%$ se obtiene que en el primer rango (a) se encuentra el tratamiento cinco (descabece del primer botón floral en punto de corte ruso más desyeme de brotes axilares) con un ciclo promedio de 127,48 días. Compartiendo los rangos a y b, se encuentra el tratamiento 4 (agobio de la

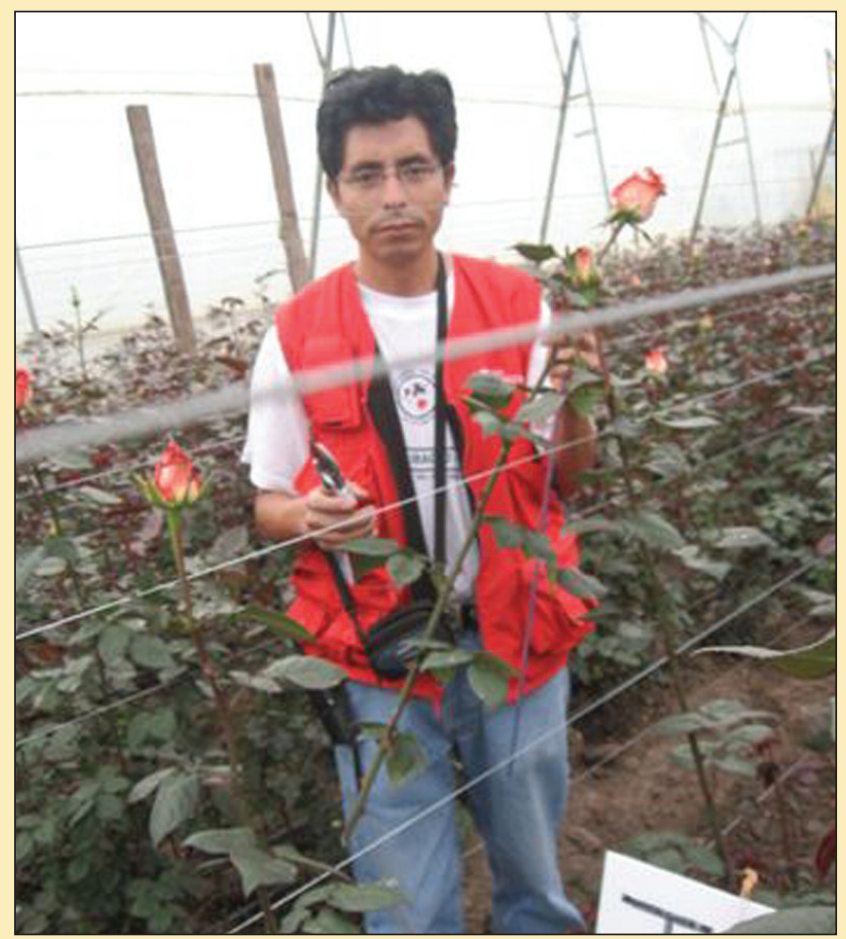

Figura 10. Cosecha de basales. Fuente: Hugo Vinueza Mejía. 


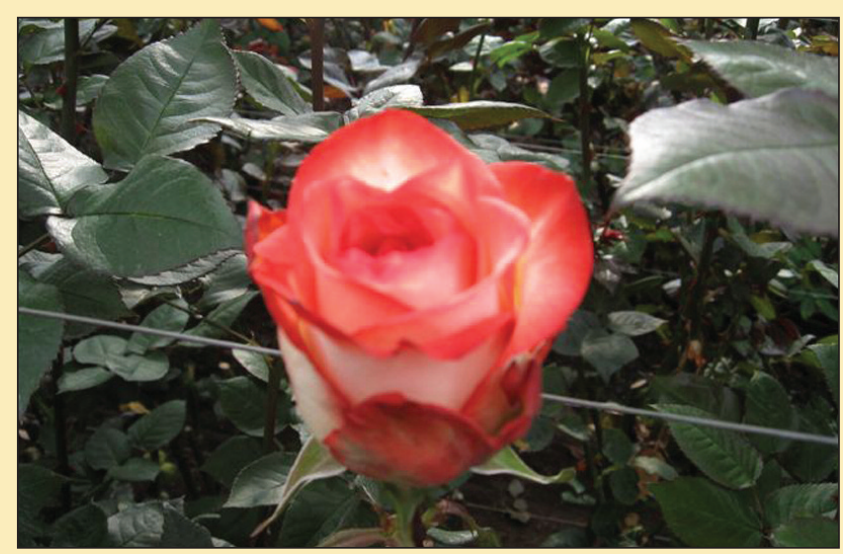

Figura II. Punto de corte ruso.

Fuente: Hugo Vinueza Mejía.

plántula por arqueo, previo descabece del primer botón floral en punto garbanzo) con un promedio de I29,88 días. La Figura 10 muestra la cosecha de los basales en punto de corte ruso.

El tratamiento 3 (cosecha de la primera flor en punto de corte ruso, presentado en las Figuras II y 12 ) se encuentra en el rango $b$ con un promedio de 136,52 días.

En el último rango (c) se localizan los tratamientos dos (agobio de la plántula por arqueo, previa formación de escobilla) y uno (agobio de la plántula con semiquiebre, previa formación de escobiIla) con un promedio de 149,4 । y 152,76 días respectivamente.

Al hacer un análisis de estos resultados, observamos que hay diferencia significativa en el ciclo de cultivo de los basales de los 5 tratamientos, evaluados desde el momento de la "siembra" de las plántulas, hasta la cosecha de los basales emitidos, debido a que desde la "siembra" de las plántulas hasta la ejecución de los tratamientos hay una variación importante en la duración de los mismos.

Tabla 5. Días a la cosecha (ciclo del cultivo) desde la ejecución de cada uno de los tratamientos

\begin{tabular}{|c|c|c|c|c|}
\hline 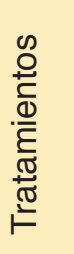 & $\begin{array}{l}\text { Promedio en días } \\
\text { a la cosecha } \\
\text { por tratamiento }\end{array}$ & ㅇำ & 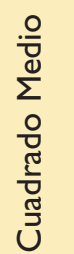 & $\vec{ن}$ \\
\hline T5 & 85,48 & a & $* *$ & 3,76 \\
\hline T3 & 94,52 & $b$ & & \\
\hline T2 & 96,41 & $b$ & & \\
\hline T4 & 97.88 & $b$ & & \\
\hline TI & 99,76 & b & & \\
\hline
\end{tabular}

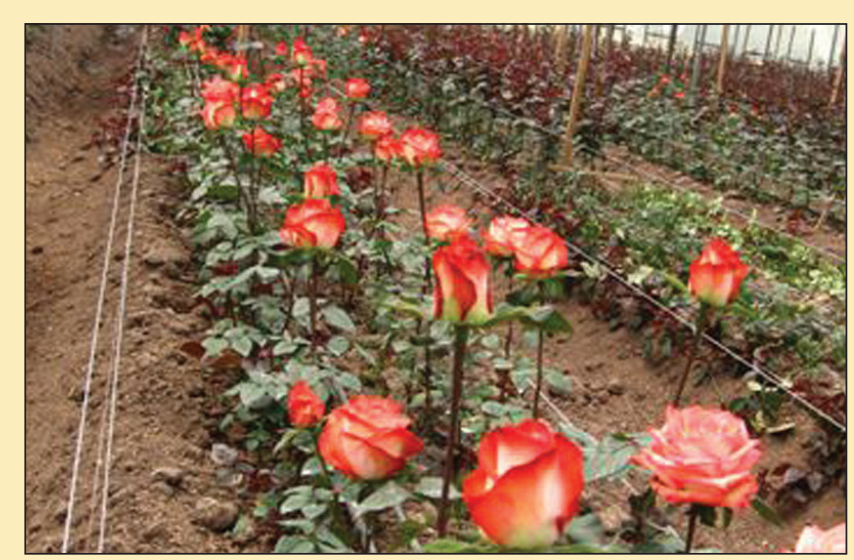

Figura 12. Tratamiento con punto de corte ruso.

Fuente: Hugo Vinueza Mejía.

En los resultados obtenidos se observa una alta significancia estadística para tratamientos con un coeficiente de variación de 3,76 que da confiabilidad a los resultados.

Aplicada la prueba de Tukey se observa que en el primer rango (a) se encuentra el tratamiento 5 con un promedio de 85,48 días. Compartiendo el segundo rango (b), se encuentran los tratamientos I, 2, 3, y 4 , es decir son iguales, en cuanto al ciclo de cultivo de los basales se refiere.

Es el tratamiento 5 el que mejor respuesta presenta respecto a los demás tratamientos, ya que tiene el menor ciclo de cultivo, con un promedio de 85,48 días.

\section{Conclusiones}

De los métodos de manejo evaluados, los mejores resultados se obtuvieron con el agobio por arqueo. Al descabezar un botón en punto de corte ruso, los basales obtenidos en este tratamiento no cumplen con los estándares de calidad requeridos en esta variedad. En lo que se refiere a la longitud de basales, los mejores resultados muestran los tratamientos 2 (agobio de la plántula por arqueo, previa formación de escobilla) y 4 (agobio de la plántula por arqueo, previo descabece del primer botón floral en punto garbanzo) en los cuales se realiza el agobio por arqueo.

Hay una relación directa entre la longitud y el calibre (diámetro) de los basales de la variedad 'Blush de los Andes', ya que los tratamientos que presentaron las mejores longitudes, mostraron también los mejores valores de diámetro, es decir en los tratamientos 2 y 4; por lo que se los considera los de mejor utilidad por ha/mes después de realizado el análisis económico. 


\section{Agradecimientos}

Un agradecimiento especial a todo el personal de la Universidad Politécnica Salesiana en Cayambe, así como al personal de la empresa florícola Rose Connection, quienes hicieron posible que se lleve adelante esta investigación.

\section{Referencias}

Duys, D.y Schouten. 200I.J. Handbook for modern greenhouse rose cultivation. Applied Plant Research. Aalsmeer y Naaldwijk, Holanda.

Ferrer F.y P. Salvador 1986. La producción de rosas en cultivo protegido. Ed. Universal Plantas S.A. España.
López P., D. Neisa,V. Flórez R. y C. Bacca. 2007. Evaluación agronómica de los sustratos espuma termoexpandible y cascarilla de arroz en el cultivo de la rosa. Floricultura Andina. En línea: http://encolombia.com/economia/floriculturandina_rosa2.htm. Consulta: 3 de febrero de 2009.

Rizzo Pastor, Pablo. 200I. Las Flores del Ecuador. Servicio de Información Agropecuaria SICA. Ministerio de Agricultura del Ecuador. Quito, Ecuador. En línea:<http://www.sica.gov.ec/agronegocios/Biblioteca/Ing\%20Rizzo/nuevos\%20exportables/flores/flores_ecuador.htm. Consulta: 20 de febrero de 2009.

Vásquez E., y Sinesio Torres 200I. Fisiología vegetal. Ciudad de la Habana-Cuba, 452 pp. 